\title{
PRODUÇÃO DE ETANOL POR LEVEDURA FLOCULANTE EMPREGANDO COMO SUBSTRATO OS RESÍDUOS POLPA E CASCAS DE BANANA MADURA
}

\author{
M. R. PADOAN ${ }^{1}$, S. A. HOPFNER ${ }^{3}$, M. S. MONTAGNOLI², N. SELLIN ${ }^{2,3}$, C. \\ MARANGONI ${ }^{3}$, O. SOUZA SO $^{1,2,3,4}$
}

Universidade da Região de Joinville - UNIVILLE, ${ }^{1}$ Departamento de Engenharia Ambiental e Sanitária, ${ }^{2}$ Departamento de Engenharia Química, ${ }^{3}$ Mestrado em Engenharia de Processos,

${ }^{4}$ Departamento de Engenharia de Produção

E-mail para contato: ozair.souza@univille.br

\begin{abstract}
RESUMO - A produção de etanol por Saccharomyces cerevisae ATCC 26603 utilizando os resíduos in natura polpa e cascas de banana como substrato de fermentação foi analisada em duas diferentes concentrações iniciais de açúcares redutores $(\mathrm{AR}=10$ e $70 \mathrm{~g} / \mathrm{L})$ e com diferentes tempos de cultivo do inóculo (18 e 24 h). Os ensaios foram realizados em frascos de Erlenmeyer de $250 \mathrm{~mL}$ contendo $80 \mathrm{~mL}$ de mosto e $20 \mathrm{~mL}$ de inóculo com pH inicial 4,5, frequência de agitação $100 \mathrm{~min}^{-1} \mathrm{e}$ temperatura de cultivo de $30^{\circ} \mathrm{C}$. A maior produtividade em etanol $\left(\mathrm{Q}_{\mathrm{P}}=2,47 \mathrm{~g} / \mathrm{L} . \mathrm{h}\right)$ foi obtida com o uso de inóculo $24 \mathrm{~h}$ e licor concentrado de cascas de banana (AR=70 $\mathrm{g} / \mathrm{L})$, com respectivo fator de conversão de substrato em produto de $0,44 \mathrm{~g} / \mathrm{g}$. Ao empregar a polpa e o licor não-concentrado de cascas $(\mathrm{AR}=6,5 \mathrm{~g} / \mathrm{L})$ como substrato foram obtidos valores médios de eficiência alcoólica $\left(\mathrm{R}_{\mathrm{P}}\right)$ da ordem de 96,6\% e 97,8\%; os quais foram maiores do que aquele alcançado com o licor concentrado de cascas $\left(\mathrm{R}_{\mathrm{P}}=86,5 \%\right)$.
\end{abstract}

\section{INTRODUÇÃO}

A necessidade de encontrar fontes alternativas de energia é cada vez mais visível no mundo atual. A escassez do petróleo, juntamente com a eficiência das energias limpas, vem promovendo ações na maioria dos países para que as energias alternativas renováveis tenham participação cada vez significativa nas matrizes energéticas.

Segundo Cortez (2008), analisando as tecnologias das fontes energéticas alternativas renováveis, suficientemente desenvolvidas para ser empregada comercialmente, somente a biomassa, utilizada em processos modernos com elevada eficiência tecnológica, possui a flexibilidade de suprir o setor energético tanto para a produção de energia elétrica quanto para mover o setor de transportes (produção de bio-óleo e bioetanol).

Do ponto de vista energético, para fim de outorga de empreendimentos do setor elétrico, biomassa é todo recurso renovável oriundo de matéria orgânica (de origem animal ou vegetal) que pode ser utilizada na produção de energia (ANEEL, 2005). 
Para a produção de etanol a partir de biomassa vegetal, os resíduos lignocelulósicos normalmente necessitam ser submetidos ao pré-tratamento químico e/ou enzimático para a conversão dos seus polímeros celulose e hemicelulose nos seus principais monômeros glicose e xilose, respectivamente. Algumas biomassas, como vários rejeitos agroindustriais de frutas, muitas vezes não precisam desses tratamentos prévios para a sua fermentação, visto já possuírem, quando maduras, grande quantidade de açúcares livres na sua composição, principalmente sacarose. Operações simples como a trituração seguida da solubilização desse açúcar em água com eventual necessidade de aumentar a sua concentração por evaporação controlada muitas vezes são suficientes para a obtenção de um mosto de características desejadas para a fermentação, ou seja, grande quantidade de açúcares fermentescíveis disponíveis e presença mínima de contaminantes capazes de inibir o processo produtivo.

Um dos maiores produtores de frutas do Brasil tem sido o Estado de Santa Catarina, onde se destacam as culturas da maçã e da banana. Segundo dados do Centro de Socieconomia e Planejamento Agrícola do estado (CEPA, 2011), nas últimas safras de 2007 a 2011, Santa Catarina foi o $3^{\circ}$ maior produtor de bananas do País contribuindo com uma produção anual média em torno de $641 \mathrm{t}$ da fruta; sendo que o litoral norte do Estado tem concentrado $85 \%$ dessa produção, predominando a espécie Musa cavendischii dos cultivares nanica e nanicão. Considerando que para cada tonelada de bananas colhidas aproximadamente $100 \mathrm{~kg}$ da fruta não são aproveitadas para consumo humano e que das frutas industrializadas cerca de $440 \mathrm{~kg}$ de cascas/t de frutas são descartadas (Souza et al., 2010), é possível estimar a geração de mais de $280 \mathrm{t}$ de resíduos por ano na região considerada. Parte dessa biomassa compõe o lixo doméstico e acaba sendo depositada em aterros sanitários, porém o restante normalmente encontra-se na indústria de processamento e nos postos de comercialização da fruta.

Em trabalho recente, Souza et al. (2012) avaliaram o uso de polpa e cascas de banana previamente tratados com diferentes concentrações de $\mathrm{H}_{2} \mathrm{SO}_{4}(0,1$ e $2 \% \mathrm{~m} / \mathrm{m})$, temperatura $\left(90,100\right.$ e $\left.120{ }^{\circ} \mathrm{C}\right)$ e tempo de residência (15 e $\left.30 \mathrm{~min}\right)$ como substrato da fermentação alcoólica por Saccharomyces cerevisae, não-floculante, isolada de fermento de panificação Em comparação ao uso das biomassas sem esse tipo de tratamento químico, os autores constataram que o emprego do ácido proporcionou um ganho percentual máximo na concentração de açúcares redutores (AR) da ordem de $8 \%$ para a polpa e de apenas $1 \%$ para as cascas. Nas condições experimentais avaliadas, o uso de $250 \mathrm{~g} / \mathrm{L}$ de massa úmida de cada um dos resíduos possibilitou a obtenção de, no máximo, $59 \mathrm{~g} / \mathrm{L}$ de $\mathrm{AR}$ para a polpa e $3 \mathrm{~g} / \mathrm{L}$ para as cascas. Segundo os autores, esses valores estão bem abaixo daquele normalmente empregado nos processos industriais de produção de etanol a partir do caldo de cana-deaçúcar (100-130 g/L). A partir dessas análises, os autores recomendaram o uso desses resíduos in natura (sem tratamento químico), triturados e dissolvidos em água através do seu cozimento a $120^{\circ} \mathrm{C} / 15 \mathrm{~min}$.

O objetivo deste trabalho foi avaliar método de extração de açúcar proposto por Souza et al. (2012) para os resíduos polpa e cascas de banana madura e, para o caso das cascas, a influência do aumento de AR inicial no mosto (concentração por evaporação) sobre a produção de etanol em processo descontínuo simples. A influência do inoculo previamente cultivado durante 18 e $24 \mathrm{~h}$ sobre a produtividade em etanol também foi avaliada neste trabalho; sendo que, para a fermentação foi empregada cultura pura de Saccharomyces cerevisae ATCC 26603, uma levedura floculante. 
O uso de micro-organismo floculante com alto rendimento na conversão de substrato em produto e com boa tolerância às altas concentrações de açúcar e etanol é desejado para a produção em escala industrial por contribuir para o seu uso no caso de processo em batelada repetida com ciclos ou no cultivo com alta densidade celular, entre outros.

\section{MATERIAIS E MÉTODOS}

\subsection{Biomassa (substrato)}

Foram avaliados dois tipos de rejeitos in natura da industrialização da banana Musa cavendishii, popularmente conhecida na região sul do Brasil como banana nanica: polpa e cascas de banana. Cada uma das biomassas foi previamente cortadas em pedaços de $1 \mathrm{a} 2 \mathrm{~cm}$ de comprimento e submetidas à trituração em liquidificador doméstico com adição de água destilada para uma concentração final de $500 \mathrm{~g} / \mathrm{L}$ de massa úmida de substrato, correspondentes à 149, $6 \mathrm{~g} / \mathrm{L}$ de massa seca para a polpa e 57,0 g/L para as cascas, conforme Souza et al. (2012).

No licor de polpa foram adicionados os nutrientes para fermentação (extrato de levedura, $3 \mathrm{~g} / \mathrm{L}$; (NH4) ${ }_{2} \mathrm{SO}_{4}, 0,5 \mathrm{~g} / \mathrm{L} ; \mathrm{K}_{2} \mathrm{HPO}_{4}, 0,5 \mathrm{~g} / \mathrm{L} ; \mathrm{MgSO}_{4}, 0,1 \mathrm{~g} / \mathrm{L}$ e $\mathrm{CaCl}_{2}, 0,1 \mathrm{~g} / \mathrm{L}$, conforme Schulz (2010) seguido por esterilização do caldo em autoclave elétrica a $120{ }^{\circ} \mathrm{C}$ durante $15 \mathrm{~min}$. Para o licor de cascas foi realizado esse mesmo tipo de procedimento, exceto que, antes da adição dos nutrientes, foi realizado o aquecimento da mistura para auxiliar na solubilização dos açúcares seguido da filtração em tecido de algodão para retenção dos sólidos suspensos. A partir do filtrado de cascas, dois tipos de caldo foram fermentados: sem concentração e com concentração prévia dos açúcares a $70{ }^{\circ} \mathrm{C}$ por evaporação simples em reator químico aberto para a atmosfera.

\subsection{Ensaios de fermentação}

Foram conduzidos em frascos Erlenmeyer de $250 \mathrm{~mL}$ contendo $100 \mathrm{~mL}$ de volume de trabalho utilizando agitador orbital, Shaker Incubating da LOGEN, na freqüência de agitação de $120 \mathrm{~min}^{-1}, 30{ }^{\circ} \mathrm{C} \mathrm{e} \mathrm{pH}$ inicial de 4,5. Como inóculo foi utilizado $20 \mathrm{~mL}(20 \% \mathrm{v} / \mathrm{v}) \mathrm{de}$ cultura pura de cepas de Saccharomyces cerevisae ATCC 26603 fornecida pela Fundação André Tosello sob número de identificação CCT 0293, previamente cultivada durante 18 ou 24 h. A manutenção da cepa foi realizada através do cultivo de superfície em meio sólido (20 g/L de Ágar-agar) empregando Placas de Petri e repiques quinzenais. O meio de cultivo utilizado tanto para a manutenção quanto para a produção de inóculo foi aquele recomendado por Schulz (2010) empregando a glicose anidra (20 g/L) como principal fonte de carbono. Foram realizados quatro ensaios de fermentação empregando os resíduos polpa e cascas: (1) Ensaio P1: polpa, inóculo 18 h de cultivo; (2) Ensaio P2: polpa, inóculo 24 h de cultivo; (3) Ensaio C1: cascas, licor não-concentrado, inóculo 24 h de cultivo e (4) Ensaio C2: cascas, licor concentrado, inóculo $24 \mathrm{~h}$ de cultivo. Como ensaio controle, ou testemunha (ensaio T) foi fermentado o mesmo meio sintético da produção do inoculo com uma concentração de 100 $\mathrm{g} / \mathrm{L}$ de glicose. 


\subsection{Métodos analíticos}

Durante as fermentações foram coletadas amostras periódicas (6 em $6 \mathrm{~h}$ ) para as determinações da concentração de açúcares redutores (AR) através do método SomogyiNelson (Somogyi, 1944; Nelson, 1952) e de etanol (P) por cromatografia gasosa (CG) utilizando cromatógrafo Agilent 6890 equipado com coluna capilar Agilent modelo HP1 (50 $\mathrm{m} \times 0,32 \mathrm{~mm} \times 1,05 \mu \mathrm{m}$, crosslinked methyl siloxane). Como gás de arraste foi empregado gás He com fluxo de $2,2 \mathrm{~mL} / \mathrm{min}$.

\subsection{Cálculo dos parâmetros cinéticos da fermentação}

Os valores de rendimento, eficiência e produtividade em etanol foram calculados de acordo com as Equações 1, 2 e 3, respectivamente.

$$
\begin{aligned}
& Y_{P / S}=\frac{\left(P_{f}-P_{0}\right)}{\left(A R_{O}-A R_{f}\right)} \\
& R_{P}=\frac{Y_{P / S}}{Y_{P / S, \text { teórico }}} 100 \\
& Q_{P}=\frac{P_{f}-P_{0}}{t_{f}}
\end{aligned}
$$

$Y_{P / S}-$ fator de conversão de substrato em produto $(\mathrm{g} / \mathrm{g})$

$R_{P}$ - rendimento percentual ou eficiência alcoólica (\%)

$Q_{P}-$ produtividade em etanol $(\mathrm{g} / \mathrm{L} . \mathrm{h})$

$P_{f}$ - concentração de etanol em $t_{f}(g / L)$

$P_{0}-$ concentração de etanol no início do processo fermentativo $(\mathrm{g} / \mathrm{L})$

$A R_{0}$ - concentração inicial de açúcares redutores no início da fermentação $(\mathrm{g} / \mathrm{L})$

$A R_{f}$ - concentração de açúcares redutores em $t_{f}(\mathrm{~g} / \mathrm{L})$

$Y_{P / S \text {,teórico }}$ valor estequiométrico da conversão bioquímica de glicose em etanol $=0,5111 \mathrm{~g} / \mathrm{g}$ $t_{f}$-tempo final de fermentação $(h)$ correspondente ao ponto de término do acúmulo de etanol

\subsection{Análise estatística}

As duplicatas dos ensaios de fermentação foram analisadas pelo método ANOVA com teste de Tukey para $\mathrm{p}<0,05$ empregando-se o programa computacional Origin 7.5.

\section{RESULTADOS E DISCUSSÃO}

Os valores das concentrações de açúcares redutores (AR) e de etanol (P) ao longo do tempo de fermentação para cada um dos resíduos avaliados podem ser vistos na Figura 1.

Em todos os ensaios realizados foi possível observar que todo o açúcar redutor disponível no início da fermentação foi praticamente exaurido até $20 \mathrm{~h}$ de cultivo, mostrando que não houve limitação do consumo do substrato por outro nutriente. Convém lembrar que 
em todos os ensaios, inclusive naquele em que foi realizado a concentração prévia do licor (Figura 1d) a concentração inicial de nutrientes foi sempre a mesma.

Comparando o uso dos diferentes tempos de cultivo do inóculo (18 e 24 h) para um mesmo tipo de composição de mosto (polpa), verifica-se que o uso de $24 \mathrm{~h}$ (Figura 1b) necessitou de menor tempo de fermentação para o consumo total de AR disponível no meio de fermentação. Este comportamento difere do observado por Schulz (2010) que ao estudar o cultivo de $S$. cerevisae não-floculante isolada de fermento comercial de padeiro, no mesmo tipo de meio sintético, indicou $18 \mathrm{~h}$ como o tempo ideal de obtenção do inóculo visto ter tido o mesmo comportamento do inóculo $24 \mathrm{~h}$.

O uso de 24 h ou até mesmo de 48 h na obtenção de inóculo de $S$. cerevisae para obtenção do inoculo em condições operacionais semelhantes a utilizada neste trabalho tem sido freqüentemente utilizado por vários autores (Siqueira et al., 2008; Groenestijn \& Slomp, 2011; Li et al., 2011; Yadav et al., 2011; Singh et al., 2012; Suriyachai et al., 2013; entre outros), contudo, em muitos casos, sem justificativa pela escolha.
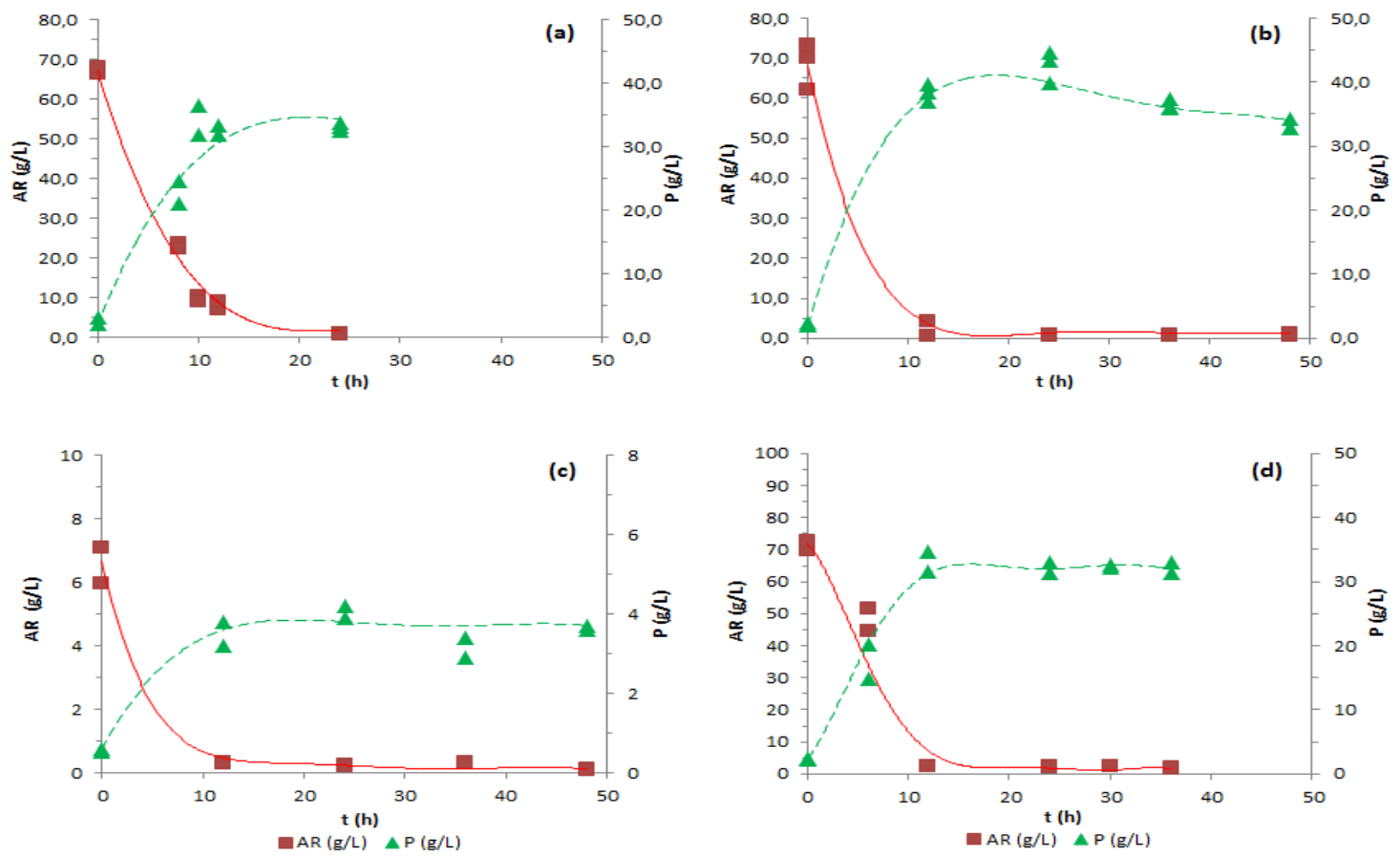

Figura 1 - Cinética do consumo de açúcares redutores $(\square \mathrm{AR})$ e produção de etanol $(\Delta \mathrm{P})$ empregando como substrato a polpa (a, b) e cascas (c, d) de banana madura com diferentes tempos de cultivo de inóculo (18 h: a; 24 h: b, c, d), sem concentração prévia do licor de fermentação (a, b e c) e com licor pré-concentrado (d).

Conforme pode ser observado na Figura 1, onde cada ponto experimental representa o valor médio obtido na análise da amostra, apenas no caso do inoculo 18h (Figura 1a), a concentração de AR se encontrava acima de $5 \mathrm{~g} / \mathrm{L}$ em $t=12 \mathrm{~h}$. Esse comportamento refletiu no tempo final de fermentação do ensaio $\left(t_{f}=20 \mathrm{~h}\right)$ o qual foi maior que nos demais 
experimentos que fizeram uso de inoculo $24 \mathrm{~h}$, exceto para o caso da glicose (Ensaio T, comportamento cinético não apresentado) conforme mostra a Tabela 1. Como o emprego de inóculo $24 \mathrm{~h}$ (Ensaio P2) apresentou maior produtividade $\left(\mathrm{Q}_{\mathrm{P}}=2,11 \mathrm{~g} / \mathrm{L} . \mathrm{h}\right)$ do que aquele resultado de inóculo $18 \mathrm{~h}$ (Ensaio P1; $\mathrm{Q}_{\mathrm{P}}=1,53 \mathrm{~g} / \mathrm{L} . \mathrm{h}$ ), o tempo de cultivo $24 \mathrm{~h}$ foi estabelecido como padrão para a realização dos demais experimentos (Ensaios C1, C2 e T).

Através do Teste de Tukey $(\mathrm{p}<0,05)$ foi possível constatar que apenas entre os valores médios dos fatores de conversão de substrato em etanol não houve diferença significativa entre a fermentação de glicose (Ensaio T), da polpa 18h (Ensaio P1) e do licor concentrado de cascas (Ensaio C2). O valor médio de $\mathrm{Y}_{\mathrm{P} / \mathrm{S}}$ para esses ensaios foi de $0,45 \pm 0,03 \mathrm{~g} / \mathrm{g}$, o qual foi menor do que aqueles obtidos nos ensaios $\mathrm{P} 2$ e $\mathrm{C} 1$, os quais mostraram eficiência alcoólica em torno de $100 \%$.

Tabela 1 - Valores médios de tempo final de fermentação $\left(t_{f}\right)$, concentração inicial $\left(\mathrm{AR}_{0}\right) \mathrm{e}$ final $\left(\mathrm{AR}_{\mathrm{f}}\right)$ de açúcares redutores, concentração inicial $\left(\mathrm{P}_{0}\right)$ e final $\left(\mathrm{P}_{\mathrm{f}}\right)$ de etanol e respectivos

parâmetros cinéticos de fator de conversão de $\mathrm{AR}$ em $\mathrm{P}\left(\mathrm{Y}_{\mathrm{P} / \mathrm{S}}\right)$, rendimento percentual ou eficiência alcoólica $\left(R_{P}\right)$ e produtividade em etanol $\left(Q_{P}\right)$ obtidos na fermentação dos resíduos polpa (Ensaios P1 e P2) e cascas de banana (Ensaio C1 e C2), e do meio sintético contendo 100 g/L de glicose (testemunha, Ensaio T); empregando como inóculo S. cerevisae ATCC 26603.

\begin{tabular}{|l|c|c|c|c|c|c|c|c|}
\hline & \multicolumn{7}{|c|}{ Variáveis do processo e respectivos parâmetros cinéticos } \\
\hline Ensaio* & $\begin{array}{c}\mathrm{t}_{\mathrm{f}} \\
(\mathrm{h})\end{array}$ & $\begin{array}{c}\mathrm{AR}_{0} \\
(\mathrm{~g} / \mathrm{L})\end{array}$ & $\begin{array}{c}\mathrm{ARf} \\
(\mathrm{g} / \mathrm{L})\end{array}$ & $\mathrm{P}_{0}(\mathrm{~g} / \mathrm{L})$ & $\mathrm{P}_{\mathrm{f}(\mathrm{g} / \mathrm{L})}$ & $\begin{array}{c}\mathrm{Y}_{\mathrm{P} / \mathrm{S}} \\
(\mathrm{g} / \mathrm{g})\end{array}$ & $\begin{array}{c}\mathrm{R}_{\mathrm{P}} \\
(\%)\end{array}$ & $\begin{array}{c}\mathrm{Q}_{\mathrm{P}} \\
(\mathrm{g} / \mathrm{L} . \mathrm{h})\end{array}$ \\
\hline $\mathrm{P} 1$ & 20 & 67 & 3 & 2,5 & 33 & 0,48 & 93,3 & 1,53 \\
\hline $\mathrm{P} 2$ & 18 & 70 & 1,2 & 2 & 40 & 0,51 & 100 & 2,11 \\
\hline $\mathrm{C} 1$ & 12 & 6,5 & 0,1 & 0,6 & 3,8 & 0,50 & 97,8 & 0,27 \\
\hline $\mathrm{C} 2$ & 12 & 68 & 2 & 2,4 & 32 & 0,44 & 86,5 & 2,47 \\
\hline $\mathrm{T}$ & 26 & 90 & 0,6 & 2,6 & 41 & 0,43 & 84,1 & 1,48 \\
\hline
\end{tabular}

* P1: inoculo previamente cultivado durante $18 \mathrm{~h} ; \mathrm{P} 2$ : inoculo $24 \mathrm{~h} ; \mathrm{C} 1$ : inóculo $24 \mathrm{~h}$, licor sem concentração prévia antes da fermentação; C2: inóculo 24 h, licor concentrado; T: inoculo 24 h, glicose 100 g/L.

Ao concentrar o licor de cascas de banana antes da fermentação (Ensaio C2) foi possível obter um valor de $\mathrm{AR}_{0}$ de $68 \mathrm{~g} / \mathrm{L}$ (Tabela 1), que fermentado resultou em uma concentração final de etanol de $32 \mathrm{~g} / \mathrm{L}$, o qual foi 8,4 vezes maior do que aquela obtida na fermentação do licor não concentrado (Ensaio $\mathrm{C} 1 ; \mathrm{P}_{\mathrm{f}}=3,8 \mathrm{~g} / \mathrm{L}$ ). Uma maior concentração de produto no caldo fermentado favorece a obtenção de maior rendimento nas operações de extração e purificação do etanol em função de menor volume de mistura a ser tratada para a obtenção de uma mesma quantidade do produto, principalmente na etapa de destilação.

Nas condições experimentais avaliadas, o aumento de $\mathrm{AR}_{0}$ no licor de cascas através da evaporação direta da mistura (evaporação de simples efeito) com conseqüente aumento de $\mathrm{P}_{\mathrm{f}}$, apesar de apresentar valores de $Y_{P / S}$ e $R_{P}$ relativamente menores, conduziu a uma produtividade em etanol de 2,47 g/L.h. Esse valor foi 52,5\% maior do que $Q_{P}$ resultado da fermentação do meio sintético empregando como principal fonte de carbono a glicose (Ensaio T). A possibilidade de aumentar ainda mais o valor de $\mathrm{AR}_{0}$ através da concentração do licor 
por evaporação parece ser uma alternativa bastante atraente para a continuidade dos estudos; contudo, faz-se necessário, também, a realização de um balanço de energia desse processo em comparação ao não concentrado, assim como uma análise econômica detalhada para estabelecer o impacto dessa operação sobre o custo final do produto.

Schulz (2010), ao empregar diferentes valores de massa úmida de cascas de banana (250 a 650 g/L), sem concentração prévia do licor, obteve valor máximo de AR de 19,8 g/L e estimou valores máximos de Yp/s e Qp da ordem de 0,40 g/g e 1,32 g/L.h, respectivamente. Esses valores foram menores do que os obtidos neste trabalho com a concentração prévia do licor de cascas (Tabela 1, Ensaio C2) demonstrando que, nas condições operacionais avaliadas, o aumento de $\mathrm{AR}_{0}$ favoreceu o processo produtivo. Novos experimentos com valores ainda maiores do que o empregado no Ensaio C2 precisam ser realizados visando a maximização de $\mathrm{Q}_{\mathrm{P}}$ sem, contudo, reduzir significativamente o rendimento e a produtividade do processo fermentativo.

\section{CONCLUSÃO}

O uso de S. cerevisae ATCC 26603 como inóculo, previamente cultivado durante $24 \mathrm{~h}$, resultou em maior produtividade em etanol na fermentação da polpa de bananas quando comparado ao inóculo $18 \mathrm{~h}$, indicando esse tempo de cultivo $(24 \mathrm{~h})$ como o ideal para a sua obtenção.

A produção de etanol a partir da fermentação de caldo de polpa de bananas com concentração de açúcares redutores em torno de $70 \mathrm{~g} / \mathrm{L}$ apresentou valor médio de fator de conversão de substrato em produto, eficiência alcoólica e produtividade em etanol semelhantes aos obtidos com o uso da glicose $100 \mathrm{~g} / \mathrm{L}$ como principal fonte de carbono.

O uso da concentração prévia do licor de cascas de banana, após solubilização dos açúcares por aquecimento, proporcionou produtividade em etanol nove vezes maior do que aquela alcançada com o licor não concentrado; comprovando, assim, a contribuição da operação de concentração sobre a produção de etanol, com concentração final no caldo fermentado de 32 g/L, ou seja, oito vezes maior do que a obtida sem a concentração.

\section{AGRADECIMENTOS}

Os autores agradecem a FAPESC - Fundação de Amparo à Pesquisa e Inovação do Estado de Santa Catarina pelo apoio financeiro.

\section{REFERÊNCIAS}

ANEEL - Agência Nacional de Energia Elétrica. Biomassa. In: Atlas de Energia Elétrica do Brasil, 2a Edição, Brasília, 2005, p. 77. Disponível em http://www.aneel.gov.br/aplicacoes/atlas/pdf/05-Biomassa\%282\%29.pdf. Acessado em 12 de abril de 2013. 
CEPA - Centro de Socieconomia e Planejamento Agrícola, Epagri, Governo do Estado de Santa Catarina. Síntese Anual da Agricultura de Santa Catarina, 2010-2011. http://cepa.epagri.sc.gov.br/Publicacoes/Sintese_2011/sintese\%202010-2011.pdf. Acessado em 14/04/2013.

CORTEZ, L.A.B.; LORA, E.E.S.; AYARZA, J.A.C. Biomassa no Brasil e no mundo. In: Cortez, L.A.B.; Lora, E.E.S.; Gómez, E.O. (Organização), Biomassa para Energia, Editora Unicamp, Campinas, 2008, p.16

GROENESTIIN, J.W.; SLOMP, R.S. Production of ethanol from wheat straw by pretreatment and fermentation at high dry matter concentrations. Industrial Biotechnology, v. 7, n. 2, p. 136-142, 2011.

LI, Y.; PARK, J.; SHIROMA, R.; TOKUYASU, K. Bioethanol production from rice straw by a sequential use of Saccharomyces cerevisiae and Pichia stipites with heat inactivation of Saccharomyces cerevisiae cells prior to xylose fermentation. J. Biosci. Bioeng., v. 111, n. 6, p. 682-686, 2011.

NELSON, N. A photometric adaptation of Somogy method for determination of glucose. Biochemistry, v. 153, p. 375-380, 1944.

SCHULZ, M.A. Produção de bioetanol a partir de rejeitos da bananicultura: polpa e cascas de banana. Dissertação de mestrado em engenharia de processos, Universidade da Região de Joinville - Univille, Joinville, Brasil, 2010, 102 p.

SINGH, A.; BISHNOI, N.R. Enzymatic hidrolysis optimization of microwave álcali pretreated wheat straw and etanol production by yeast. Bioresour. Technol., v. 108, p. 94-101, 2012.

SIQUEIRA, P.F.; KARP, S.G.; CARVALHO, J.C.; STURM, W.; RODRIGUEZ-LEON, J.A.; THOLOZAN, J.; SINGHANIA, R.R.; PANDEY, A.; SOCCOL, C.R. Production of bioethanol from soybean molasses by Saccharomyces cerevisiae at laboratory, pilot and industrial scales. Bioresour. Technol., v. 99, p. 8156-8163, 2008.

SOMOGYI, M. Notes on sugar determination. J. Biol. Chem., v. 195, p. 19, 1952.

SOUZA, O.; FEDERIZZI, M.; COELHO, B.; WAGNER, T. M.; WISBECK, E. Biodegradação de resíduos lignocelulósicos gerados na bananicultura e sua valorização para a produção de biogás. Rev. bras. eng. agríc., v. 14, n. 4, p. 438-443, 2010.

SOUZA, O.; SCHULZ, M.A.; FISCHER, G.A.A.; WAGNER, M.T.; SELLIN, N. Energia alternative de biomassa: bioetanol a partir da casca e da polpa de banana. Rev. bras. eng. agríc., v. 16, n. 8, p. 915-921, 2012.

SURIYACHAI, N.; WEERASAIA, K.; LAOSIRIPOJANA, N.; CHAMPREDA, V.; UNREAN, P. Optimized simultaneous saccharification and co-fermentation of rice straw for ethanol production by Saccharomyces cerevisiae and Scheffersomyces stripits co-culture using design of experiments. Bioresour. Technol., v. 142, p. 171-178, 2013.

YADAV, K.S.; NASEERUDDIN, S.; PRASHANTHI, G.S.; SATEESH, L.; RAO, L.V. Bioethanol fermentation of concentrated rice straw hydrolysate using co-culture of Saccharomyces cerevisiae and Pichia stipitis. Bioresour. Technol., v. 102, p. 6473-6478, 2011. 